PROCEEDINGS OF THE AMERICAN MATHEMATICAL SOCIETY

Volume 124, Number 7, July 1996

\title{
K-THEORY AND THE ANTI-AUTOMORPHISM OF THE STEENROD ALGEBRA
}

\author{
M. C. CRABB, M. D. CROssley, AND J. R. HUBBUCK
}

(Communicated by Eric Friedlander)

\begin{abstract}
We give simple proofs of some relations in the Steenrod algebra involving the powers $\mathcal{P}^{i}$ and their duals $\chi \mathcal{P}^{i}$ and show how these relations
\end{abstract} arise from $K$-theory.

\section{INTRODUCTION}

Barratt and Miller in [3] derived some relations in the Steenrod algebra $\mathcal{A}(p)$ using expressions for the Adem relations due to Bullett and Macdonald [4]. In Section 2 we reformulate and give a simple proof of the former in the spirit of the latter. These relations arise naturally from properties of the Adams operations in complex $K$-theory, as was explained in $[7,6]$ for the prime 2 ; alternative and shorter $K$-theoretic derivations valid for all primes are given in Section 3.

\section{Results of Barratt and Miller}

We use standard notation for the $\bmod p$ Steenrod algebra $\mathcal{A}(p)$ with the convention that $\mathcal{P}^{i}$ denotes $S q^{i}$ when $p=2$. For each non-negative integer $n$, let

$$
\phi(n)=\min \left\{k \geq 0 \mid k+\nu_{p}(k !) \geq n\right\} .
$$

Another, more illuminating description of $\phi(n)$ is given in Proposition 2.6.

Theorem 2.1. The polynomial

$$
F_{n}(T)=\sum_{i=0}^{n} \mathcal{P}^{n-i} \chi\left(\mathcal{P}^{i}\right) T^{i} \in \mathcal{A}(p)[T]
$$

is divisible by $(T-1)^{\phi(n)}$.

This result can be restated in many ways. Let $T^{m} F_{n}(T)$, for $m \in \mathbb{Z}$, be expressed as a formal power series in $S=T-1$. By considering the coefficient of $S^{j}$ with $0 \leq j<\phi(n)$, it follows that

$$
\sum_{i=0}^{n}\left(\begin{array}{c}
m+i \\
j
\end{array}\right) \mathcal{P}^{n-i} \chi\left(\mathcal{P}^{i}\right)=0 \quad \text { for } 0 \leq j<\phi(n) .
$$

Looking at $T^{n} F\left(T^{-1}\right)$ instead of $F(T)$ (or applying the anti-automorphism $\chi$ ) we obtain

$$
\sum_{i=0}^{n}\left(\begin{array}{c}
m+i \\
j
\end{array}\right) \mathcal{P}^{i} \chi\left(\mathcal{P}^{n-i}\right)=0 \quad \text { for } 0 \leq j<\phi(n) .
$$

Received by the editors January 31, 1995.

1991 Mathematics Subject Classification. Primary 55S10; Secondary 55N15.

(C)1996 American Mathematical Society 
This is Theorem 1 of [3]. (The condition $0 \leq j<\phi(n)$ is equivalent to $(p-1) n>$ $j p-\alpha(j)$ in the notation of [3] and setting $(N, K, L)=(n, j-m-1, j)$ gives $\left.\left(\begin{array}{c}m+i \\ j\end{array}\right)=(-1)^{L}\left(\begin{array}{c}K-i \\ L\end{array}\right).\right)$

It will be convenient to write $\gamma(r)=\left(p^{r}-1\right) /(p-1)$.

Corollary 2.4. Let $n \geq \gamma(r+1)-r$ and $0 \leq k<p^{r}$. Then

$$
\sum_{j \geq 0} \mathcal{P}^{k+p^{r} j} \chi\left(\mathcal{P}^{n-k-p^{r} j}\right)=0 .
$$

This is Theorem 3.2 of [3]. The condition $n \geq \gamma(r+1)-r$ is equivalent to $p^{r} \leq \phi(n)$; see Proposition 2.6. From Theorem 2.1, $F_{n}(T)$ is divisible by $T^{p^{r}}-1=$ $(T-1)^{p^{r}}$ and the result follows as a polynomial $\sum a_{i} T^{i}$ is divisible by $T^{N}-1$ if and only if $\sum a_{k+N j}=0$ for each $k, 0 \leq k<N$.

From Corollary 2.4, one obtains Straffin's formula [10]:

$$
\sum_{j=0}^{p} \mathcal{P}^{p^{r} j} \chi\left(\mathcal{P}^{p^{r}(p-j)}\right)=0 .
$$

In the next proposition we collect some properties of $\phi(n)$. (We are grateful to the referee for drawing our attention to the characterization $(d)$ of $\phi$.)

Proposition 2.6. The function $\phi$ has the following properties.

(a) $\phi(\gamma(r))=p^{r-1}$, for $r \geq 1$.

(b) $\phi(i+j) \leq \phi(i)+\phi(j)$ for $i, j \geq 0$.

(c) Let $\gamma(r)<n<\gamma(r+1)$. Then $\phi(n)=\phi(n-\gamma(r))+\phi(\gamma(r))$.

(d) Let $\psi(n)$, defined for non-negative integers $n$, satisfy: $\psi(0)=1 ; \psi(i+j) \leq$ $\psi(i)+\psi(j)$ for $i, j \geq 0 ; \psi(\gamma(r)) \leq p^{r-1}$ for $r \geq 1$. Then $\psi(n) \leq \phi(n)$ for all $n \geq 0$.

Proof. Recall that $\nu_{p}(k !)=\left(k-\alpha_{p}(k)\right) /(p-1)$, where $\alpha_{p}(k)$ is the sum of the coefficients in the $p$-adic expansion of $k$. One finds that $p^{r-1}+\nu_{p}\left(p^{r-1} !\right)=\gamma(r)$, and this establishes (a).

Part (b) follows from the inequality $k+\nu_{p}(k !)+l+\nu_{p}(l !) \leq(k+l)+\nu_{p}((k+l) !)$.

In (c) we have $p^{r-1}<\phi(n) \leq p^{r}$. Consider $k=\phi(n)-p^{r-1}$. Now

$$
\alpha_{p}(k)=\alpha_{p}(\phi(n))+ \begin{cases}-1 & \text { if } \phi(n)<p^{r} \\ p-2 & \text { if } \phi(n)=p^{r}\end{cases}
$$

and

$$
k+\nu_{p}(k !)=\phi(n)+\nu_{p}(\phi(n) !)-\gamma(r)-\left\{\begin{array}{ll}
0 & \text { if } \phi(n)<p^{r} \\
1 & \text { if } \phi(n)=p^{r}
\end{array} .\right.
$$

In both cases we have $k \geq \phi(n-\gamma(r))$, since $n<\gamma(r+1)$ when $\phi(n)=p^{r}$. The reverse inequality is supplied by $(b)$.

Part (d) is then an immediate consequence of (a)-(c).

The properties of $\psi$ postulated in (d) above are precisely those required of $\phi$ in the proof of Theorem 2.1 which follows.

Proof of Theorem 2.1. Both the hypothesis and the conclusion of the theorem are multiplicative: $\phi$, as we have noted above, is subadditive and the $F_{n}(T)$ satisfy a Cartan formula. For let $P=\sum_{i \geq 0} \mathcal{P}^{i} w^{i} \in \mathcal{A}(p)[[w]]$ be the total Steenrod power and $P_{T}^{-1}=\sum_{i \geq 0} \chi\left(\mathcal{P}^{i}\right)(w T)^{i}$ in $\mathcal{A}(p)[T][[w]]$. So $P \cdot P_{T}^{-1}=\sum_{n \geq 0} F_{n}(T) w^{n}$. As both $P$ and its inverse are multiplicative on cohomology classes, so is $P \cdot P_{T}^{-1}$. 
It is, therefore, sufficient to verify the assertion in the theorem on a generator $e \in H^{2}\left(\mathbb{C} P^{\infty} ; \mathbb{F}_{p}\right)$ for $p$ odd or $e \in H^{1}\left(\mathbb{R} P^{\infty} ; \mathbb{F}_{2}\right)$ for $p=2$. (See, for example, Chapters I and VI of [9].)

A short calculation shows that

$$
\left(P \cdot P_{T}^{-1}\right) e=\sum_{r \geq 0}(-1)^{r} e^{p^{r}}\left(1+w e^{p-1}\right)^{p^{r}}(w T)^{\gamma(r)} .
$$

So

$$
F_{n}(T) e= \begin{cases}e, & n=0, \\ (-1)^{r} e^{p^{r}}(T-1)^{p^{r-1}} T^{\gamma(r-1)}, & n=\gamma(r), r \geq 1, \\ 0, & \text { otherwise. }\end{cases}
$$

Since $\phi(\gamma(r))=p^{r-1}$, this completes the proof.

The proof of Theorem 1 in [3] uses an auxiliary result which can be established by similar reasoning.

Proposition 2.7. The polynomial

$$
G_{n}(T)=\sum_{i=0}^{n} \chi\left(\mathcal{P}^{n-i}\right) \mathcal{P}^{i} T^{i} \in \mathcal{A}(p)[T]
$$

is of the form $(T-1)^{r} g\left(T^{p}\right)$, where $r \equiv n(\bmod p)$.

Again statements are multiplicative and it suffices to check the result on the class $e$. One verifies that

$$
G_{n}(T) e= \begin{cases}e, & n=0 \\ (-1)^{r} e^{p^{r}}(1-T), & n=\gamma(r), r \geq 1, \\ 0, & \text { otherwise. }\end{cases}
$$

So, setting $S=T-1$ as above and expanding $T^{p m} G_{n}(T)$, it follows that

$$
\sum_{i=0}^{n}\left(\begin{array}{c}
i+p m \\
j
\end{array}\right) \chi\left(\mathcal{P}^{n-i}\right) \mathcal{P}^{i}=0, \quad \text { when } j \not \equiv n(\bmod p) .
$$

The basic technique in these proofs can be found already in work of Atiyah and Hirzebruch [2]. Working in the category of finite CW-complexes we set

$$
\mathcal{V}^{*}(-)=H^{*}\left(-; \mathbb{F}_{p}\right) \otimes \mathbb{F}_{p}[w],
$$

where $w$ has degree -1 if $p=2$ and $-2(p-1)$ if $p$ is odd, and equip the cohomology theory $\mathcal{V}^{*}$ with the obvious product.

Any automorphism $A$ of this multiplicative theory which fixes $w$ is of the form

$$
A=\sum_{i \geq 0} \alpha_{i} w^{i}, \quad \text { with } \alpha_{i} \in \mathcal{A}(p), \text { and } \alpha_{0}=1 .
$$

Now $A(e)=\sum_{r \geq 0} a_{r} e^{p^{r}} w^{\gamma(r)}, a_{r} \in \mathbb{F}_{p}$, with $a_{0}=1$. (As we have restricted attention to finite complexes, we should work with the skeleta of the infinite-dimensional projective space.) We associate to $A$ the formal power series

$$
\xi_{A}(X)=\sum_{r \geq 0} a_{r} w^{\gamma(r)} X^{p^{r}} \in \mathbb{F}_{p}[w][[X]], \quad a_{0}=1,
$$

and notice that $\xi_{A B}=\xi_{B} \circ \xi_{A}$. 
Theorem 2.10 (Atiyah-Hirzebruch). The map $A \mapsto \xi_{A}(X)$ gives an anti-isomorphism between the group of automorphisms of the multiplicative cohomology theory $\mathcal{V}^{*}(-)$ which fix $w$ and the group of formal power series of the form

$$
\sum_{r \geq 0} a_{r} w^{\gamma(r)} X^{p^{r}} \in \mathbb{F}_{p}[w][[X]], \quad a_{0}=1 .
$$

The basic example of such an automorphism is the total Steenrod power $P$. More generally, given a commutative $\mathbb{F}_{p}$-algebra $R$, one can consider $R$-automorphisms of the cohomology theory $\mathcal{V}^{*}(-) \otimes R$. Theorem 2.10 generalizes immediately. We have been working with the polynomial ring $R=\mathbb{F}_{p}[T]$.

For reference we write

$$
\pi(X)=\xi_{P}(X), \quad \pi_{T}(X)=\xi_{P_{T}}(X) .
$$

\section{Connections with Adams operations in $K$-theory}

The arithmetic result used to translate statements about complex $K$-theory with $p$-local coefficients into statements about cohomology operations with $\mathbb{F}_{p^{-}}$ coefficients is the following.

Lemma 3.1. Let $f(T) \in \mathbb{Z}_{(p)}[T]$ be a polynomial such that $p^{n}$ divides $f\left(k^{p-1}\right)$ for every integer $k$ prime to $p$. Then the $\bmod p$ reduction of $f(T)$ in $\mathbb{F}_{p}[T]$ is divisible by $(T-1)^{\phi(n)}$.

Proof. We recall that when $g(U) \in \mathbb{Q}[U]$ is a rational polynomial of degree $d$ which has the property that $g(l) \in \mathbb{Z}_{(p)}$ for every $l \in \mathbb{Z}$, then for some $b_{i} \in \mathbb{Z}_{(p)}$

$$
g(U)=\sum_{i=0}^{d} b_{i}\left(\begin{array}{c}
U \\
i
\end{array}\right)
$$

Given (non-zero) $f(T)$ of degree $d$, we define $g(U)=p^{-n} f(1+p U)$. If $k$ is prime to $p$, then $k^{p-1}=1+p l$ for some $l \in \mathbb{Z}$. On the other hand, for any integer $l$ there is some $k$ prime to $p$ with $k^{p-1} \equiv 1+p l\left(\bmod p^{n}\right)$. So $g(l) \in \mathbb{Z}_{(p)}$ for all $l \in \mathbb{Z}$. Hence

$$
f(T)=\sum_{i=0}^{d} p^{n} b_{i}\left(\begin{array}{c}
(T-1) / p \\
i
\end{array}\right)
$$

for some $b_{i} \in \mathbb{Z}_{(p)}$.

By comparing coefficients of $T^{d}$, we see that $p^{n-d-\nu_{p}(d !)} b_{d}$ is equal to the leading coefficient $a_{d} \in \mathbb{Z}_{(p)}$ of $f(T)$. So

$$
p^{n} b_{d}\left(\begin{array}{c}
(T-1) / p \\
d
\end{array}\right) \in \mathbb{Z}_{(p)}[T]
$$

and has $\bmod p$ reduction $a_{d}(T-1)^{d}$. If $d<\phi(n)$, then $a_{d}$ is divisible by $p$. Otherwise, there is no restriction on $a_{d} \bmod p$. The proof is completed by induction on $d$.

We note that for each integer $n \geq 0$ there is a polynomial $f(T)$ satisfying the hypotheses of Lemma 3.1 with $\bmod p$ reduction equal to $(T-1)^{\phi(n)}$, namely

$$
f(T)=p^{k} k !\left(\begin{array}{c}
(T-1) / p \\
k
\end{array}\right)
$$

where $k=\phi(n)$. So the exponent $\phi(n)$ in Lemma 3.1 cannot be improved. 
Until the final paragraph of this section we will now assume that $p$ is an odd prime. We give two closely related ways of seeing that the relations of Theorem 2.1 arise from standard properties of Adams operations.

The first follows the approach of $[7,6]$. For a finite CW-complex $Z$ without homology $p$-torsion, it was shown in [8], by pulling the unstable Adams operations apart, that there exist homomorphisms $S^{i}: H^{*}\left(Z ; \mathbb{Z}_{(p)}\right) \rightarrow H^{*+2(p-1) i}\left(Z ; \mathbb{Z}_{(p)}\right)$, with $S^{0}$ the identity, satisfying certain properties. (These operations are not natural: their construction depends upon a choice of isomorphism between $K$-theory and cohomology.) When reduced $\bmod p, S^{i}$ coincides with the Steenrod power $\mathcal{P}^{i}$. Let us introduce

$$
\Phi_{n}(T)=\sum_{i=0}^{n} S^{n-i} \chi\left(S^{i}\right) T^{i}
$$

where $\chi\left(S^{i}\right)$ is defined inductively by $\sum_{i=0}^{n} S^{n-i} \chi\left(S^{i}\right)=0$ if $n>0$ and is the identity homomorphism if $n=0$. Corollary 2.12 of [8] asserts that the homomorphism $\Phi_{n}\left(k^{p-1}\right)$ is divisible by $p^{n}$, as a linear operator, for each integer $k$ prime to $p$.

Now Lemma 3.1 extends immediately to the polynomial $f(T)=\Phi_{n}(T)$ with values in the endomorphism ring of $H^{*}\left(Z ; \mathbb{Z}_{(p)}\right)$. Hence $F_{n}(T)$ is divisible by $(T-1)^{\phi(n)}$ as an endomorphism of $H^{*}\left(Z ; \mathbb{F}_{p}\right)$. Since relations among the Steenrod powers are detected by torsion-free spaces, Theorem 2.1 follows.

The second approach is closer to that in Section 2. Let $\ell^{*}$ be the Adams summand of $p$-local connective complex $K$-theory. Again restricting attention to finite complexes, we define a multiplicative cohomology theory $\mathcal{W}^{*}$ by

$$
\mathcal{W}^{*}(-)=H^{*}\left(-; \mathbb{Z}_{(p)}\right) \otimes \mathbb{Z}_{(p)}[w]=\bigoplus_{j \geq 0} H^{*+2(p-1) j}\left(-; \mathbb{Z}_{(p)}\right) w^{j}
$$

where $w$ has degree $-2(p-1)$. Both the Adams integral Chern character

$$
\operatorname{ch}: \ell^{*}(-) \rightarrow \mathcal{W}^{*}(-)
$$

and the Thom homomorphism $\operatorname{dim}: \ell^{*}(-) \rightarrow H^{*}\left(-; \mathbb{Z}_{(p)}\right)$ are multiplicative transformations. We have $\operatorname{ch}(v)=p w$, where $v$ is a Bott generator of $\ell^{-2(p-1)}$.

According to Adams there is the commutative diagram:

(See [1] and, for example, [5].)

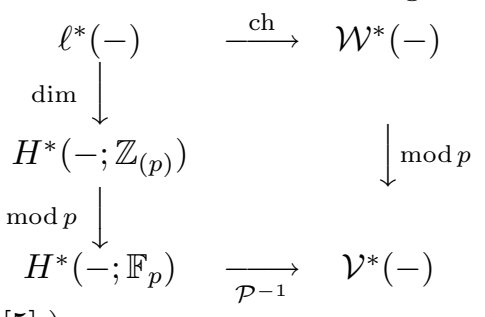

We choose a complex orientation for $\ell^{*}$ lifting the standard orientation on integral cohomology. Let $e_{\ell}(\lambda) \in \ell^{2}(Z)$ be the associated Euler class of a complex line bundle $\lambda$ over a finite complex $Z$ and $e(\lambda) \in H^{2}\left(Z ; \mathbb{Z}_{(p)}\right)$ be the standard Euler class.

By looking at the Hopf line bundle over $\mathbb{C} P^{\infty}$ we see that

$$
\operatorname{ch}\left(e_{\ell}(\lambda)\right)=\sigma^{-1}(e(\lambda)),
$$

for some formal power series $\sigma^{-1}(X)=\sum t_{n} w^{n} X^{(p-1) n+1}$, in $\mathbb{Z}_{(p)}[w][[X]]$, with $t_{0}=1$. The inverse formal power series $\sigma(X)$ has a similar form $\sum s_{n} w^{n} X^{(p-1) n+1}$ 
with $s_{0}=1$. We shall also need $\sigma_{T}(X)=\sum s_{n}(w T)^{n} X^{(p-1) n+1}$. From the commutative diagram above one deduces that $\sigma$ and $\sigma_{T}^{-1}$ lift the power series $\pi$ and $\pi_{T}^{-1}$ in $\mathbb{F}_{p}[w][[X]]$ corresponding to the total Steenrod power and its inverse, (2.11).

The Adams operations $\psi^{k}$ are defined on $\ell^{*}(-)$ for $k$ prime to $p$. We have

$$
\operatorname{ch} \circ \psi^{k}=\psi_{\mathcal{W}}^{k} \circ \mathrm{ch},
$$

where the corresponding operator $\psi_{\mathcal{W}}^{k}$ on $\mathcal{W}^{*}(-)$ is the identity on $H^{*}\left(-; \mathbb{Z}_{(p)}\right)$ and $\psi_{\mathcal{W}}^{k}(w)=k^{p-1} w$.

In $\ell^{*}$-theory we can write $\psi^{k}\left(e_{\ell}(\lambda)\right)=\Psi^{k}\left(e_{\ell}(\lambda)\right)$, where

$$
\Psi^{k}(X)=\sum c_{n} v^{n} X^{(p-1) n+1} \in \mathbb{Z}_{(p)}[v][[X]], \quad c_{0}=1 .
$$

The relations in Theorem 2.1 arise from the equality

$$
\operatorname{ch}\left(\psi^{k}\left(e_{\ell}(\lambda)\right)=\psi_{\mathcal{W}}^{k}\left(\operatorname{ch}\left(e_{\ell}(\lambda)\right)\right) .\right.
$$

This gives

$$
\Psi_{\mathcal{W}}^{k}\left(\sigma^{-1}(e)\right)=\sigma_{k^{p-1}}^{-1}(e),
$$

where $\Psi_{\mathcal{W}}^{k}$ is obtained from $\Psi^{k}$ by substituting $p w$ for $v$. But by considering $\mathbb{C} P^{\infty}$, this is a general equality in $\mathbb{Z}_{(p)}[w][[X]]$ when $e$ is replaced by $X$, and so

$$
\Psi_{\mathcal{W}}^{k}(X)=\sigma_{k^{p-1}}^{-1}(\sigma(X)) .
$$

We consider the coefficient of $w^{n} X^{n(p-1)+1}$ in $\sigma_{T}^{-1}(\sigma(X))$, which is a polynomial $f_{n}(T) \in \mathbb{Z}_{(p)}[T]$. Now $f_{n}\left(k^{p-1}\right)$, the coefficient of $w^{n} X^{n(p-1)+1}$ in $\Psi_{\mathcal{W}}^{k}(X)$, is divisible by $p^{n}$. The reduction of $f_{n}(T) \bmod p$ is the coefficient of $w^{n} X^{n(p-1)+1}$ in $\pi_{T}^{-1}(\pi(X))$. So Lemma 3.1 applies to give a non-computational proof of the essential step in the proof of Theorem 2.1 that $F_{n}(T) e$ is divisible by $(T-1)^{\phi(n)}$.

When $p=2, \mathcal{P}^{i}$ in this section must be replaced by $S q^{2 i}$ and the cohomology theories $\mathcal{V}^{*}(-)$ and $\mathcal{W}^{*}(-)$ re-defined with the class $w$ of degree -2 . Then the results above are valid. The connection with Theorem 2.1 can be made, as in [6], by noting that a homogeneous polynomial in the $S q^{2 i}$ vanishes on the cohomology of a product of complex projective spaces if and only if the same polynomial in the $S q^{i}$ vanishes on the corresponding product of real projective spaces.

Remarks on formal groups. The formal power series occurring in Theorem 2.10 as the automorphisms of the cohomology theory $\mathcal{V}^{*}(-)$ are the strict automorphisms of the additive formal group law $F$ over $\mathbb{F}_{p}[w]: F(X, Y)=X+Y$. (The degree of $X$ is 1 if $p=2$ and 2 if $p$ is odd.) The ring of endomorphisms of $F$ is the ring $\mathbb{F}_{p}[[\mathcal{F}]]$ of formal power series in the Frobenius $\mathcal{F}, \mathcal{F}(X)=X^{p}$. The automorphism group is the group of units in this ring with constant term 1 and is free abelian over (the $p$-adic integers) $\mathbb{Z}_{p}$ of rank $p-1$. More generally, if we work over a commutative $\mathbb{F}_{p}$-algebra $R$, the endomorphism ring is the twisted formal power series ring $R[[\mathcal{F}]]$, with $\mathcal{F} r=r^{p} \mathcal{F}$ for $r \in R$. The calculations in the proof of Theorem 2.1 are carried out in this ring, with $R=\mathbb{F}_{p}[T]$.

In Section 3, the power series $\sigma$ and $\sigma^{-1}$ are isomorphisms between the additive formal group law $F$ over $\mathbb{Z}_{(p)}[w]$ and the group law $F_{\ell}$ coming from the chosen complex orientation of $\ell^{*}$ :

$$
F(\sigma(X), \sigma(Y))=\sigma(X)+\sigma(Y)=\sigma\left(F_{\ell}(X, Y)\right) .
$$




\section{REFERENCES}

1. J. F. Adams, On the Chern character and the structure of the unitary group, Proc. Cambridge Philos. Soc. 57, (1961), 189-199. MR 22:12525

2. M. F. Atiyah and F. Hirzebruch, Cohomologie-Operationen und charakteristische Zahlen, Math. Zeit. 77, (1961), 149-187. MR 27:6285

3. M. G. Barratt and H. R. Miller, On the Anti-automorphism of the Steenrod Algebra, Contemp. Math. 12, (1981), 47-52.

4. S. R. Bullett and I. G. Macdonald, On the Adem relations, Topology 21, (1982), 329-332. MR 83h:55035

5. M. C. Crabb, S. Jäschke and K. Knapp, The Im(J)-theory Chern character, manuscript, 1992.

6. M. D. Crossley, Results concerning the Steenrod Algebra, Thesis, University of Aberdeen, 1995.

7. M. D. Crossley and J. R. Hubbuck, Not The Adem Relations, Bol. Soc. Mat. Mexicana 37, (1992), 99-105.

8. J. R. Hubbuck, Primitivity in torsion free cohomology Hopf algebras, Comm. Math. Helv. 46, (1971), 13-43. MR 44:5958

9. N. E. Steenrod, Cohomology Operations, Ann. of Math. Studies 50, Princeton University Press, 1962. MR 26:3056

10. P. D. Straffin, Identities for conjugation in the Steenrod algebra, Proc. Amer. Math. Soc. 49, (1975), 235-255. MR 52:1693

Department of Mathematical Sciences, University of Aberdeen, Aberdeen AB9 2Ty, UNITED KINGDOM

E-mail address: m.crabb@maths.abdn.ac.uk

E-mail address: j.hubbuck@maths.abdn.ac.uk

Current address, M. D. Crossley: Centre de Recerca Matemàtica, Institut d'Estudis Catalans, Apartat 50 E-08193, Bellaterra (Barcelona), Spain

E-mail address: crossley@bianya.com.es 